RELATO DE CASO

\title{
Síndrome de Klippel-Trenaunay-Weber: associação do tratamento operatório à escleroterapia por espuma
}

\author{
Klippel-Trenaunay-Weber Syndrome: association of operative treatment with foam sclerotherapy \\ Ronald Luiz Gomes Flumignan', Daniel Guimarães Cacione², Silvia Iglesias Lopes 3 , Caroline Nicacio Bessa Clezar4, \\ Carolina Dutra Queiroz ${ }^{5}$, Aécio Rubens Dias Pereira-Filho ${ }^{6}$, Newton de Barros Jr? , Fausto Miranda Jr ${ }^{8}$
}

\begin{abstract}
Resumo
A Síndrome de Klippel-Trenaunay-Weber representa a associação de hemangiomas planos, ectasias venosas e hipertrofia do segmento corpóreo afetado. Apresenta-se o caso de um paciente de 39 anos, sexo masculino, seguido desde 1993 no serviço da Disciplina de Cirurgia Vascular da Escola Paulista de Medicina (Unifesp), por quadro inicial da síndrome em membro inferior esquerdo. O paciente foi tratado apenas por elastocompressão e medidas gerais. Neste período, ele evoluiu com piora da estase venosa, da hipertrofia óssea e da hiperpigmentação de pele. Na ultrassonografia Doppler colorida venosa dos membros inferiores havia perviedade do sistema venoso profundo, hiperfluxo venoso, insuficiência segmentar de veia safena magna com sinais de tromboflebite e tributárias insuficientes. Indicou-se, para a melhora da estase venosa, ligadura da junção safeno-femoral esquerda e escleroterapia (polidocanol 3\%) retrógrada da mesma, com exérese das ectasias venosas. O paciente recebeu alta hospitalar no primeiro dia pós-operatório e atualmente está em acompanhamento ambulatorial com melhora significativa dos sintomas.
\end{abstract}

Palavras-chave: Varizes; escleroterapia; Síndrome de Klippel-Trenaunay-Weber; soluções esclerosantes

\begin{abstract}
The Klippel-Trenaunay-Weber Syndrome is the association of hemangioma, venous ectasia, and hypertrophy of the affected body segment. We report the case of a 39-year-old male followed-up since 1993 due to onset of symptoms in the left lower limb. He was treated only with the use of elastic stockings and general measures. Over the years, he had worsening of venous stasis, of bone hypertrophy, and of skin hyperpigmentation. Color-coded Doppler ultrasonography of the lower limbs showed patency of the deep venous system, venous overflow, segmentar insufficiency of the greater saphenous vein with signs of thrombophlebitis, and insufficient tributary veins. In order to improve venous stasis, ligation of the left sapheno-femoral junction and retrograde foam sclerotherapy (polidocanol 3\%) with resection of tributary veins were performed. The patient was discharged in the first postoperative day and has been followed as an outpatient, presenting significant improvement of the symptoms.
\end{abstract}

Keywords: Varicose veins; sclerotherapy; Klippel-Trenaunay-Weber Syndrome; sclerosing solutions

\section{Introdução}

A Síndrome de Klippel-Trenaunay (SKT) foi descrita, em 1900, pelos médicos franceses Maurice Klippel e Paul Trenaunay e associa más-formações vasculares (hemangiomas planos e ectasias venosas) e hipertrofia do membro afetado ${ }^{1-3}$. Posteriormente, em 1907, Parkes Weber ${ }^{4}$ descreveu uma síndrome semelhante que incluía também fístulas arteriovenosas. Após ter sido identificado o aparecimento de fístulas arteriovenosas em pacientes com SKT, alguns autores tratam as duas entidades nosológicas como sendo partes de uma mesma doença, que foi chamada de Síndrome de Klippel-Trenaunay-Weber (SKTW) $)^{2,5,6}$, sendo que outros preferem ainda tratar separadamente $e^{3,7,8}$.

A causa exata da SKT não é bem conhecida, porém, sugere-se que seja decorrente de alteração do mesoderma

\footnotetext{
${ }^{1}$ Médico Cirurgião Vascular, Doutorando da Universidade Federal de São Paulo (Unifesp) - São Paulo (SP), Brasil.

${ }^{2}$ Médico Cirurgião Vascular da Unifesp - São Paulo (SP), Brasil.

${ }^{3}$ Médica Cirurgiã Vascular da Unifesp - São Paulo (SP), Brasil.

${ }^{4}$ Médica Cirurgiã Vascular, Mestranda da Unifesp - São Paulo (SP), Brasil.

${ }^{5}$ Médica Cirurgiã Vascular, Doutoranda da Unifesp - São Paulo (SP), Brasil.

${ }^{6}$ Médico Cirurgião Vascular da Unifesp - São Paulo (SP), Brasil.

${ }^{7}$ Docente da Disciplina de Cirurgia Vascular da Unifesp - São Paulo (SP), Brasil.

${ }^{8}$ Professor Titular da Disciplina de Cirurgia Vascular da Unifesp - São Paulo (SP), Brasil.

Submetido em: 02.12.10. Aceito em: 15.03 .11

J Vasc Bras. 2011;10(1):77-80.
} 
durante o desenvolvimento embrionário ${ }^{6,8}$. Especula-se que haja alteração ao nível do antagonista da angiopoetina- $2^{8}$, com manutenção de pequenas comunicações arteriovenosas nos membros que provocarão a sintomatologia associada $^{2}$, embora alguns autores postulem que a hipertrofia de tecidos moles é alteração primária e não se relaciona às fístulas ${ }^{6}$.

A presença dos sintomas e sua intensidade podem variar desde a ocorrência de hemangiomas planos discretos até ectasias venosas de grande volume e grande deformidade dos membros ${ }^{9}$. Somente $63 \%$ dos pacientes diagnosticados com a SKTW apresentam os três sinais clássicos de malformações, hemangiomas planos e ectasias venosas com hipertrofia do segmento afetado ${ }^{2,8,10}$. Um total de $95 \%$ dos pacientes apresenta acometimento das extremidades, prevalecendo as inferiores. A grande maioria dos pacientes (98\%) apresenta hemangiomas planos cutâneos ${ }^{2,7,9,10}$. Grandes dilatações venosas superficiais e profundas são comuns na SKTW e podem piorar com a idade e com a progressão da doença pelo refluxo venoso complexo, incompetência valvular, hipertensão venosa e prejuízo da função de bomba muscular9.

Aproximadamente $50 \%$ dos pacientes são tratados de maneira conservadora com auxílio de elastocompressão e não necessitam de tratamento cirúrgico ${ }^{7,9,10}$. Alguns pacientes com veias profundas pérvias e competentes se beneficiam da escleroterapia, ressecção escalonada de veias varicosas e exérese dos aneurismas venosos ${ }^{8,10}$.

As principais causas de dor no membro afetado estão relacionadas com as anormalidades da insuficiência venosa crônica, tais como: celulite, tromboflebite superficial, trombose venosa profunda, calcificação da malformação vascular e malformações vasculares intraósseas ${ }^{10}$.

\section{Relato de caso}

O presente trabalho está de acordo com a Resolução nº 196/96 do Conselho Nacional de Saúde, de 10 de outubro de 1996.

Paciente de 39 anos, auxiliar de almoxarifado, natural de Alagoas, procedente de São Paulo, procurou o Serviço de Cirurgia Vascular da Escola Paulista de Medicina da Universidade Federal de São Paulo em 1993, com queixa de varizes na perna esquerda. Nesta ocasião, ele relatou a presença de veias varicosas que aumentaram de calibre desde a infância. Aos 17 anos, começou a apresentar manchas avermelhadas no membro, principalmente ao redor das veias varicosas. Não apresentava outros antecedentes pessoais ou familiares. Nessa ocasião, iniciou tratamento conservador com elastocompressão, hidratação de pele e controle das infecções cutâneas.

Em 2000, o paciente retornou ao ambulatório com piora das varizes, hiperpigmentação de face medial de perna esquerda (CEAP C 4a) $)^{11-15}$, hemangiomas planos e aumento do comprimento do membro inferior esquerdo (MIE) por hipertrofia óssea (escanograma com diferença de $2,5 \mathrm{~cm}$ no comprimento do fêmur). Os pulsos arteriais eram simétricos e normais. Foi mantido com tratamento clínico e, em 2006, houve aparecimento de grande ectasia venosa $(5 \mathrm{~cm})$ na região anterior do joelho esquerdo (Figura 1). Outros membros da família do paciente foram investigados e não foi encontrado um sinal ou sintoma clínico de síndrome semelhante em nenhum deles ${ }^{16,17}$.

Com a evolução da doença, surgiram novas ectasias venosas (Figura 2), de aproximadamente $5 \mathrm{~cm}$, na região da coxa, joelho e perna esquerda. Foram relatados episódios de tromboflebites superficiais tratadas por analgesia e anti-inflamatórios não- esteroides. Em abril de 2009, a ultrassonografia Doppler colorida (UDC) revelou sistema venoso profundo pérvio, sem sinais de trombos recentes ou antigos. A UDC evidenciou ainda hiperfluxo nas veias femorais comum, superficial e profunda, sugerindo presença de microfístulas arteriovenosas. No sistema superficial havia insuficiência segmentar de veia safena magna desde $13 \mathrm{~cm}$ abaixo da prega inguinal até tornozelo, com sinais de tromboflebite prévia e tributária insuficientes.

Em razão da sintomatologia dolorosa, dos episódios de tromboflebite e da limitação das atividades físicas e laborais, indicou-se tratamento operatório para desconexão safenofemoral (SF) por ligadura da junção SF e realização de escleroterapia com microespuma de polidocanol a 3\%, injetada em cateter introduzido retrogradamente pelo coto distal da safena magna alcançando até o nível do joelho ${ }^{18}$. Além disso, realizou-se a exérese das ectasias venosas na coxa, joelho e perna esquerdas e esclerose com polidocanol a 3\% dos ramos remanescentes (Figura 3) por injeção direta. A microespuma foi obtida com a mistura do polidocanol a 3\% com ar ambiente numa diluição de 1:4 mL, segundo a técnica de Tessari ${ }^{19}$, com volume total de $10 \mathrm{~mL}$. O paciente apresentou boa recuperação, obteve alta hospitalar no primeiro dia pós-operatório, mantendo tratamento conservador com elastocompressão, antibioticoterapia profilática e cuidados de higiene e está em acompanhamento ambulatorial neste serviço como mostra a Figura 4.

O paciente manteve-se bem até dezembro de 2009, quando referiu reinício das dores na região do joelho esquerdo, em face lateral, na qual havia grande ectasia venosa com cerca de $4 \mathrm{~cm}$ de diâmetro (Figura 5). Optou-se por realização de nova sessão de escleroterapia com microespuma (polidocanol a 


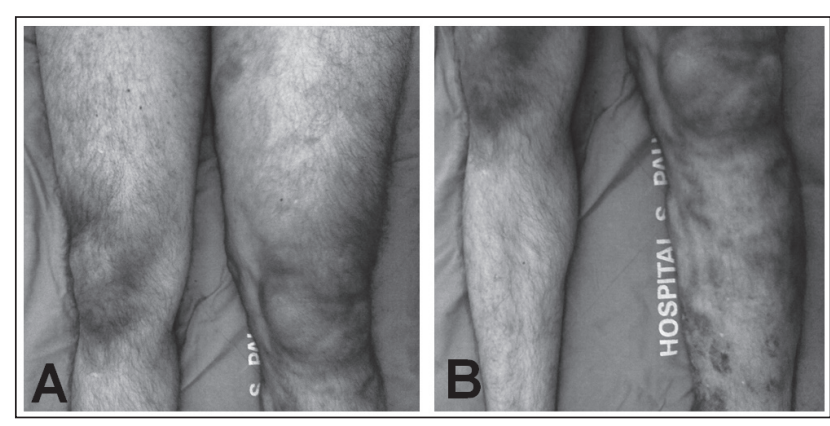

Figura 1 - Vista anterior dos membros inferiores de paciente com Síndrome de Klippel-Trenaunay-Weber. A - hipertrofia de membro inferior esquerdo desnivela a interlinha dos joelhos. B - dermatite ocre, hemangiomas planos em perna esquerda e abaulamento em região medial de joelho esquerdo (ectasia venosa)

3\%) ambulatorial, por punção direta e compressão local. No sétimo dia pós-escleroterapia, verificou-se redução importante do tamanho da ectasia venosa (Figura 5) e melhora dos sintomas. Atualmente, ele segue em acompanhamento ambulatorial com elastocompressão e cuidados locais.

\section{Discussão}

Alguns autores ${ }^{16,17}$ verificaram a ocorrência de associação familiar do aparecimento da SKTW. Neste caso, como na maioria da literatura, não há relato de ocorrência desta síndrome na família, talvez por estar relacionada à mutação autossômica dominante de penetrância incompleta ${ }^{8}$. O paciente em questão apresentou-se ao nosso serviço com quadro inicial de veias varicosas sem lesão cutânea (hemangioma plano), que só se manifestou posteriormente. Quadro esse pouco típico, uma vez que os pacientes costumam se apresentar com hemangiomas cutâneos de início, podendo progredir com ectasias venosas e, por fim, com a hipertrofia do membro afetado ${ }^{1,2,7,9,10}$.

Os autores que consideram a SKT separadamente da SKTW defendem que a presença de fístulas arteriovenosas interfere diretamente na gravidade do quadro e em seu tratamento ${ }^{8}$. Aqueles que consideram que se trata apenas de uma entidade nosológica (SKTW) justificam que estas fístulas não interferem no quadro clínico, nem tampouco na relação com hipertrofia de tecidos ${ }^{6}$. Todos os autores concordam, no entanto, é que as medidas conservadoras continuam norteando o tratamento da SKTW. Isso não afasta a necessidade de intervenções pontuais durante a evolução da história natural da doença. Estas terapias adjuvantes podem variar desde a terapia a laser, escleroterapia com microespuma, ressecções escalonadas de veias ectasiadas e até exéreses mais amplas ${ }^{6-10,15}$. As indicações mais utilizadas para o tratamento operatório são: as hemorragias, as infecções locais, o tromboembolismo e a

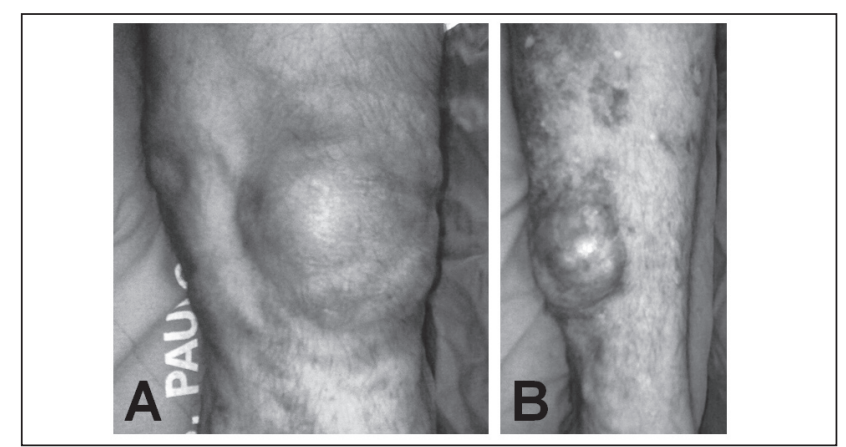

Figura 2 - Vista anterior de membro inferior esquerdo de paciente com Síndrome de Klippel-Trenaunay-Weber. A - ectasia venosa em joelho esquerdo. B - ectasia venosa em perna esquerda

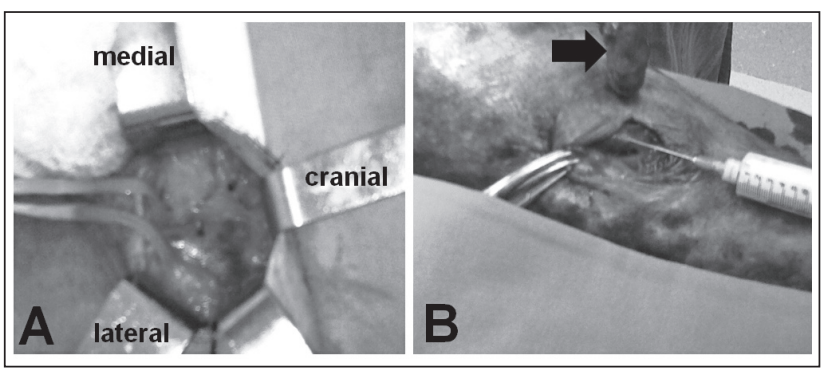

Figura 3 - Paciente com Síndrome de Klippel-Trenaunay-Weber. A região inguinal esquerda dissecada e exposta croça de safena magna (reparo com fita vascular). B - face medial de perna esquerda dissecada pós-exérese de ectasia venosa (seta). Esclerose de tributárias remanescentes com polidocanol 3\% (seringa)

ocorrência de ulcerações de perna muito refratárias. Outras indicações são: dor local, limitação funcional e estética ${ }^{8}$. No caso descrito, o sistema venoso profundo era pérvio e competente (como na maioria dos casos), permitindo a indicação do tratamento operatório ${ }^{7-10}$. A limitação funcional associada à dor local das ectasias venosas foi determinante para essa indicação cirúrgica.

A SKTW é doença rara, com morbidade progressiva e grave, deve ser tratada com parcimônia e individualização na escolha do melhor tratamento e época de realizá-lo para cada paciente. Atualmente, a indicação de intervenções operatórias é restrita às complicações decorrentes do quadro inicial. No presente caso, a utilização de associação do tratamento operatório com o escleroterápico se mostrou eficiente e conseguiu permitir uma melhora no quadro clínico e de qualidade de vida do paciente, mostrando que pode ser uma alternativa válida para estes casos. Avanços maiores serão feitos quando for possível diagnosticar ainda mais precocemente a SKTW e impedir o desenvolvimento da hipertrofia de tecidos, angiodisplasias complexas e outras alterações fenotípicas, talvez corrigindo ou impedindo a mutação genética relacionada. 


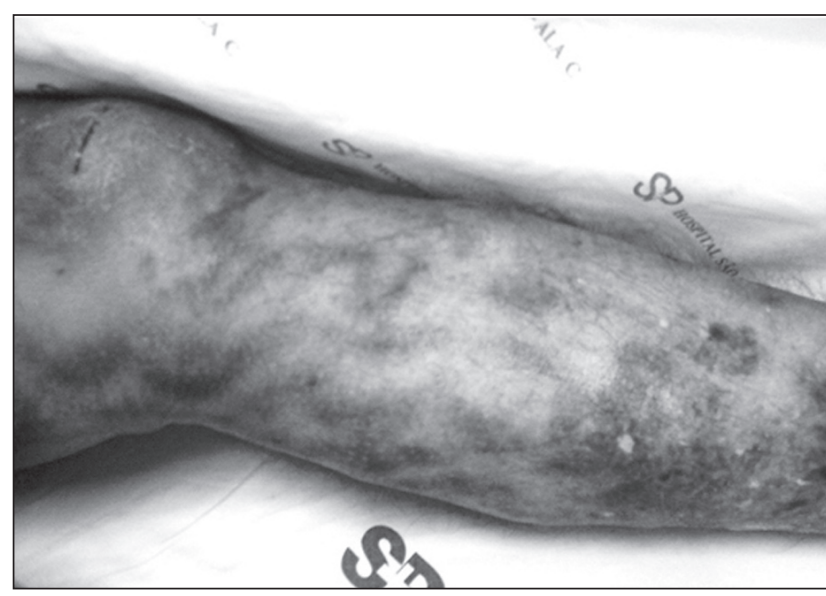

Figura 4 - Paciente com Síndrome de Klippel-Trenaunay-Weber. Face medial de perna esquerda no sétimo dia de pós-operatório

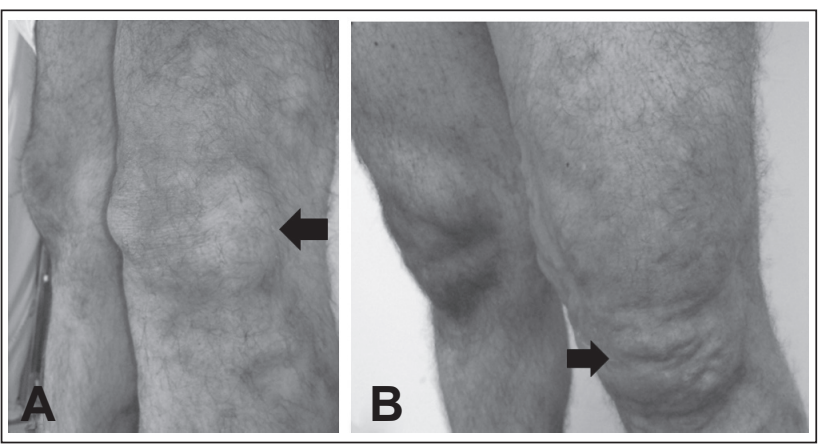

Figura 5 - Paciente com Síndrome de Klippel-Trenaunay-Weber. A - região do joelho esquerdo, em janeiro de 2010, com novo aneurisma venoso em face lateral (seta). B - região do joelho esquerdo após sete dias de escleroterapia com polidocanol 3\%. Redução importante do tamanho do aneurisma venoso (seta)

\section{Referências}

1. Klippel M, Trenaunay P. Du noevus variquex oste-hypertrophic. Arch Gen Med. 1900;185:641-72.

2. Latessa V, Frasier K. Case study: A minimally invasive approach to the treatment of Klippel-Trenaunay syndrome. J Vasc Nurs. 2007;25:76-84.

3. Campos HGA, Curado JH. Angiodisplasias. In: Maffei FHA, Lastória S, Yoshida WB, et al. Doenças Vasculares Periféricas. $4^{\mathrm{a}}$ ed. Rio de Janeiro: Guanabara Koogan; 2008. p. 1949-76.

4. Weber PF. Angioma formation in connection with hypertrophy of limbs and hemihypertrophy. Br J Dermatol. 1907;19:231-5.

5. Mullins JF, Naylor D, Redetzki J. The Klippel-Trenaunay-Weber syndrome. Arch Dermatol. 1962;86:202-6.

6. Villela ALC, Guedes LGS, Paschoa VVA, et al. Perfil epidemiológico de 58 portadores de síndrome de Klippel-Trenaunay-Weber acompanhados no Ambulatório da Santa Casa de São Paulo. J Vasc Bras. 2009;8(3):219-24.
7. Lindenauer SM. The Klippel-Trenaunay Syndrome: Varicosity, Hypertrophy and Hemangioma With No Arteriovenous Fistula. Ann Surg. 1965;162(2):303-4.

8. Gloviczki P, Driscoll DJ. Klippel-Trenaunay syndrome: current management. Phlebology. 2007;22:291-8.

9. Frasier K, Giangola G, Rosen R, et al. Endovascular radiofrequency ablation: A novel treatment of venous insufficiency in KlippelTrenaunay patients. J Vasc Surg. 2008;47:1339-45.

10. Delis KT, Gloviczki P, Wennberg PW, et al. Hemodynamic impairment, venous segmental disease, and clinical severity scoring in limbs with Klippel-Trenaunay syndrome. I Vasc Surg. 2007;45:561-7.

11. Beebe HG, Bergan JJ, Bergqvist D, et al. Classification and grading of chronic venous disease in the lower limbs. A consensus statement. Eur J Vasc Endovasc Surg. 1996;12(4):487-91.

12. Beebe HG, Bergan JJ, Bergqvist D, et al. Classification and grading of chronic venous disease in the lower limbs. A consensus statement. Int Angiol. 1995;14(2):197-201.

13. Beebe HG, Bergan JJ, Bergqvist D, et al. Classification and grading of chronic venous disease in the lower limbs--a consensus statement. Organized by Straub Foundation with the cooperation of the American Venous Forum at the 6th annual meeting, February 22-25, 1994, Maui, Hawaii. Vasa. 1995;24(4):313-8.

14. Eklöf B, Rutherford RB, Bergan Jj, et al. Revision of the CEAP classification for chronic venous disorders: Consensus statement. J Vasc Surg. 2004; 40:1248-52.

15. Vasquez MA, Munschauer CE. Venous Clinical Severity Score and quality-of-life assessment tools: application to vein practice. Phlebology. 2008;23:259-75.

16. Aelvoet G, Jorens P, Roelen L. Genetic aspects of the KlippelTrenaunay syndrome. Br J Derm. 1992;126:603-7.

17. Jorgenson R, Darby B, Patterson R, Trimmer KJ. Prenatal diagnosis of the Klippel-Trenaunay-Weber syndrome. Pren Diag. 1994;14:989-92.

18. Figueiredo M, Araújo S, Barros-Jr N, Miranda-Jr F. Results of Surgical Treatment Compared with Ultrasound-Guided Foam Sclerotherapy in Patients with Varicose Veins: A Prospective Randomised Study. Eur J Vasc Endovasc Surg. 2009;38:758-63.

19. Tessari L, Cavezzi A, Frullini A. Preliminary experience with a new sclerosing foam in the treatment of varicose veins. Dermatol Surg. 2001;27:58-60.

Correspondência: Ronald Luiz Gomes Flumignan Rua Leandro Dupret, 377, Apto 111 - Vila Mariana CEP: 04025-011 - São Paulo (SP), Brasil E-mail: flumignan@gmail.com

Contribuições dos autores: Concepção e desenho do estudo: RLGF, NBJ, FMJ Análise e interpretação dos dados: RLGF, DGC, SIL, CNBC, CDQ, ARDP, NBJ, FMJ Coleta de dados: RLGF, DGC, SIL, CNBC, CDQ, ARDP, NBJ, FMJ Redação do artigo: RLGF, NBJ, FMJ Revisão crítica do texto: RLGF, DGC, SIL, CNBC, CDQ, ARDP, NBJ, FMJ Aprovação final do artigo*: RLGF, DGC, SIL, CNBC, CDQ, ARDP, NBJ, FMJ Análise estatística: RLGF, NBJ, FMJ Responsabilidade geral pelo estudo: RLGF, DGC, SIL, CNBC, CDQ, ARDP, NB], FMJ Informações sobre financiamento: RLGF, DGC, SIL, CNBC, CDQ, ARDP, NBJ, FMJ

* Todos os autores leram e aprovaram a versão final submetida ao J Vasc Bras. 